\title{
Identification of gene biomarkers with expression profiles in patients with allergic rhinitis
}

\author{
Yun Hao ${ }^{1 \dagger}$, Boqian Wang ${ }^{1 \dagger}$, Jinming Zhao ${ }^{1 \dagger}$, Ping Wang ${ }^{1,2}$, Yali Zhao ${ }^{1,2}$, Xiangdong Wang ${ }^{1,2}$, Yan Zhao ${ }^{1,2^{*}}$ and
} Luo Zhang ${ }^{1,2,3^{*}}$ (D)

\begin{abstract}
Background: Allergic rhinitis (AR) is an upper respiratory tract inflammation disease caused by lgE-mediated reactions against inhaled allergens. The incidence of AR is significantly increasing throughout the world. Hence, more specific, and sensitive gene biomarkers and understanding the underlying pathways are necessary to further explore the AR pathogenesis.
\end{abstract}

Objective: To identify gene biomarkers in nasal mucosa and in blood from AR patients which could be used in AR diagnosis.

Methods: The gene expression profiles of GSE43523 from nasal epithelial cells and GSE75011 from Th2-enriched CD4+ T cells in blood were downloaded from the Gene Expression Omnibus database. Gene Ontology (GO), Kyoto Encyclopedia of Genes and Genomes (KEGG) analyses and protein-protein interaction (PPI) network analysis were conducted to investigate the functional changes of genes. The receiver operating characteristic (ROC) curves were used to assess the diagnostic values of the hub genes. Real-time quantitative PCR (RT-qPCR) was performed to validate the hub genes.

Results: Significant differentially enriched gene signatures in AR patients were identified in nasal epithelial cells (n-DEGs) and in blood (t-DEGs). Signatures associated with axoneme, extracellular matrix, collagen fibril organization, cell motility, calcium ion binding, and so on were more enriched in n-DEGs, whereas signatures associated with TNF signaling pathway, detoxification of inorganic compound, and cellular response to corticotropin-releasing hormone stimulus were enriched in t-DEGs. In addition, we identified 8 hub genes and 14 hub genes from n-DEGs and t-DEGs, respectively. The combination of POSTN in nasal mucosa and PENK and CDC25A in blood was constructed with a good AR predicting performance. The area under the curve (AUC) of the ROC curve of 3 hub genes' combination was 0.98 for AR diagnosis.

Conclusion: This study utilized gene expression profiles and RT-qPCR validation on nasal mucosa and blood from AR patients to investigate the potential biomarkers for AR diagnosis.

\footnotetext{
*Correspondence: zhaoyanray@126.com; dr.luozhang@139.com

†Yun Hao, Bogian Wang and Jinming Zhao contributed equally to this work

${ }^{2}$ Beijing Laboratory of Allergic Diseases and Beijing Key Laboratory of Nasal Diseases, Beijing Institute of Otolaryngology, Beijing 100005, China

Full list of author information is available at the end of the article
} permits use, sharing, adaptation, distribution and reproduction in any medium or format, as long as you give appropriate credit to the original author(s) and the source, provide a link to the Creative Commons licence, and indicate if changes were made. The images or other third party material in this article are included in the article's Creative Commons licence, unless indicated otherwise in a credit line to the material. If material is not included in the article's Creative Commons licence and your intended use is not permitted by statutory regulation or exceeds the permitted use, you will need to obtain permission directly from the copyright holder. To view a copy of this licence, visit http://creativecommons.org/licenses/by/4.0/. The Creative Commons Public Domain Dedication waiver (http://creativeco mmons.org/publicdomain/zero/1.0/) applies to the data made available in this article, unless otherwise stated in a credit line to the data. 
Keywords: Allergic rhinitis, Differentially expressed genes, Bioinformatic analysis, Biomarkers, Diagnosis

\section{Background}

Allergic rhinitis (AR) is defined as symptoms of nasal obstruction, rhinorrhea, sneezing, and nasal pruritus caused by IgE-mediated reactions against inhaled allergens and involving mucosal inflammation driven by type 2 helper $\mathrm{T}$ (Th2) cells [1]. It affects approximately 17 to $28.5 \%$ of adults in Europe and 10 to $30 \%$ of adults in the United States [2]. It has been previously shown that the prevalence of AR has increased from 11.1 to $17.6 \%$ in adults in China [3]. AR is also associated with comorbidities such as asthma, rhinosinusitis, conjunctivitis, nasal polyposis and otitis media [4]. Recent studies have demonstrated that an imbalance in innate and adaptive immunity together with environmental factors plays a key role in AR pathogenesis [5]. Serum IgE in AR may be derived from nasal mucosal production [6], suggesting that research on nasal mucosa and peripheral blood in AR patients should not be separated. Although significant advances have been made in understanding the pathophysiology of AR, its early diagnosis, therapeutic intervention, and underlying pathogenesis remain challenges. Therefore, elucidating the unique characteristics of AR is important for developing biomarkers for its diagnosis and novel clinical strategies for its treatment.

Currently, next-generation sequencing and bioinformatic analysis have emerged as promising, useful tools for screening genetic alterations involved in the development and progression of diseases, with marked clinical applications ranging from molecular diagnosis to disease classification, patient stratification to therapeutic prediction, and new drug target discovery to response prediction $[7,8]$. Previous studies have utilized samples from nasal epithelial cells, peripheral blood mononuclear cells (PBMCs) or Th2-enriched CD4+ $\mathrm{T}$ cells to identify differentially expressed genes (DEGs) in AR patients compared to healthy controls [9-11]. Although some studies have focused on the analysis of AR-related DEGs, there is a lack of an integrative analysis of local and circulating samples to elucidate the complexity of AR pathogenesis.

The utility of AR biomarkers for clinical trials depends on both sensitivity of the measure but also the practicality of the testing and sampling. Both the collection of nasal epithelial cells and peripheral blood are rapid and minimally invasive procedures. Realtime quantitative PCR (RT-qPCR), as one of the most financially accessible and widely available detection technologies is commonly used for the identification of gene biomarkers both in clinical and research studies.

In this study, we aimed to explore the possible molecular mechanisms by integrating databases on nasal epithelial cells and peripheral Th2-enriched CD4+ cells from AR patients and the same cells from healthy controls. We described the transcriptional features and identified gene biomarkers to shed light on AR development at the molecular level and to pave the way toward understanding potential pathogenesis mechanisms to facilitate its diagnosis.

\section{Methods}

\section{Ethics approval and consent to participate}

The study was approved by the Ethics Committee of Beijing TongRen Hospital, Capital Medical University in accordance with the guidelines of the World Medical Association's Declaration of Helsinki., and informed consent was obtained from all participants before the study.

A total of 20 subjects, including 10 AR patients and 10 healthy controls were recruited in this study. The diagnosis of AR was made according to the Allergic Rhinitis and its Impact on Asthma (ARIA) guidelines [12], including (1) presence of persistent or discontinuous symptoms of continuous sneezing, anterior rhinorrhoea, nasal obstruction, and itching, (2) a pale and edematous nasal mucosa, nasal discharge, and swollen inferior turbinate by nasal endoscopy, (3) positive antigen sIgE in serum that measured by the ImmunoCAP 1000 system (Pharmacia, Uppsala, Sweden). The result of the serum specific IgE consists of D1, D2 and PHAD. D1 stands for Dermatophagoides pteronyssinus, D2 stands for Dermatophagoides farinae, and PHAD stands for phadiatop, which suggests a respiratory allergy to inhalant allergens. Subjects were considered "positive" for AR diagnosis more than a cutoff of $0.35 \mathrm{kU} / \mathrm{L}$ of serum sIgE. The exclusion criteria are including: (1) co-morbid asthma, eczema, or any other allergic diseases; (2) hypertension, diabetes, or other chronic diseases; or (3) tumor in the nasal cavity or any other inflammatory nasal disease. Details of the subjects' characteristics are included in Table 1.

All subjects underwent nasal brushing of the 1 nare. The brush samples were immediately put into phosphate buffered saline containing $2 \%$ bovine serum albumin on ice, underwent RNA isolation after centrifugate at $300 g$, 
Table 1 Clinical characteristics of healthy controls and AR patients

\begin{tabular}{lllc}
\hline & AR $(\mathbf{n}=\mathbf{1 0})$ & Control $(\mathbf{n}=\mathbf{1 0})$ & P value \\
\hline Age (year, mean \pm SD) & $34.3 \pm 1.461$ & $31.6 \pm 3.121$ & 0.4435 \\
Sex (Female/Male) & $6 / 4$ & $6 / 4$ & $>0.9999$ \\
VAS (mean $\pm S D)$ & $5.475 \pm 0.7139$ & 0 & $<0.0001$ \\
PHAD (KUA/L, mean $\pm S D)$ & $9.096 \pm 3.78$ & $0.181 \pm 0.02519$ & 0.0299 \\
D1 (KUA/L, mean $\pm S D)$ & $3.412 \pm 1.963$ & $0.17 \pm 0.02556$ & 0.116 \\
D2 (KUA/L, mean $\pm S D)$ & $7.115 \pm 4.249$ & $0.148 \pm 0.02375$ & 0.1184 \\
Total IgE (KUA/L, & $383.8 \pm 142$ & $23.16 \pm 2.395$ & 0.0205 \\
mean $\pm S D)$ & & & \\
\hline
\end{tabular}

$A R$ allergic rhinitis, VAS visual analog scale, $S D$ standard deviation, $P H A D$ phadiatop

$10 \mathrm{~min}$, at $4{ }^{\circ} \mathrm{C}$. The $2 \mathrm{~mL}$ whole blood samples were collected with tubes with EDTA anticoagulant, then lysis by $10 \times$ red blood cell lysis buffer and then washed twice by phosphate buffered saline. Total RNA was extracted from nasal brush samples and blood samples according to the manufacturer's protocol of TRIzol reagent (SigmaAldrich) for real-time quantitative PCR analysis.

\section{Microarrays, datasets, and characteristics of clinical samples from the Gene Expression Omnibus (GEO) data repository}

GSE43523 (https://www.ncbi.nlm.nih.gov/geo/query/ acc.cgi?acc $=$ GSE43523), a dataset derived from nasal epithelial cells, and GSE75011 (https://www.ncbi.nlm. nih.gov/geo/query/acc.cgi?acc=GSE75011), a dataset derived from Th2-enriched CD4+ T cells from peripheral blood, were obtained from the GEO database. GSE43523, which includes seven samples from patients with seasonal AR (SAR) and five samples from non-allergic healthy controls, was derived from the GPL6883 platform (Illumina HumanRef-8 v3.0 expression bead chip), and GSE75011, which includes 25 samples from patients with AR and 15 samples from healthy controls, was derived from the GPL16791 platform (Illumina HiSeq 2500) [11]. The flow chart of our study is shown in Additional file 1: Figure S1.

\section{Differential gene expression analysis}

First, background correction and standardization were performed on the original GEO datasets using the packages edge $\mathrm{R}$ and limma of $\mathrm{R}$ software [13]. Next, differential gene analysis $\left(\left|\log _{2} \mathrm{FC}\right|>1, P\right.$-value $\left.<0.05\right)$ was performed to compare AR patients and healthy controls with the limma package of $\mathrm{R}$ software. Heatmaps and volcano plots of the differentially expressed genes (DEGs) were constructed using the packages pheatmap and ggplot2 of R software [14].

\section{Functional enrichment and pathway analyses}

We analyzed the functions and signaling pathways of the DEGs using MetaCore ${ }^{\mathrm{TM}}$ (version 20.3, Thomson Reuters, New York, NY, USA), a computational platform capable of analyzing gene clusters in the context of functional enrichment and pathway analyses. Pathway maps and Gene Ontology (GO) analyses were performed in MetaCore [15]. GO annotation includes three kinds of functional categories: biological process (BP), cellular component (CC) and molecular function (MF). Functional enrichment analysis was based on the cutoff value of $P<0.05$.

\section{Protein-protein interaction (PPI) network construction}

STRING (version 11.0) (http://string-db.org/) was used to identify the PPIs of the intersecting DEGs of the two datasets, with a combined score $>0.4$ used as the threshold for statistically significant interactions [16]. Cytoscape (version 3.7.2) software was used to further visualize the PPI network [17]. Then, the ClueGO and CluePedia plugins were used to decipher functionally grouped GO and pathway annotation networks in the PPI network [18]. A $P$-value $<0.05$ and a $\mathrm{K}$ coefficient of 0.4 were considered the threshold values. Then, the Molecular Complex Detection (MCODE) plugin was used to identify important proteins in the PPI network [19]. In addition, the degree cutoff was set as 2, the node score was set as 0.2 , the k-score was set as 2 , and the maximum depth was set as 100 . Proteins with a false discovery rate $($ FDR $)<0.05$ were considered statistically significant. The ClueGO plugin was then used to perform GO and Kyoto Encyclopedia of Genes and Genomes (KEGG) functional enrichment analyses. Kappa coefficients were calculated to reflect the functional correlations between GO terms or KEGG pathways and were defaulted to values greater than or equal to 0.4 [20].

\section{Circular visualization}

A circular layout is a convenient and efficient way to visualize large amounts of genomic information. The circlize package of $\mathrm{R}$ software was utilized to visualize relations among the chromosome locations, gene biotypes, and expression levels of the DEGs [21].

\section{Reverse transcription and real-time quantitative PCR (RT-qPCR)}

Total RNA was isolated from lysates of nasal brushing cells and white blood cells using TRIzol reagent (SigmaAldrich) according to the manufacturer's protocol. The 
concentration and purity of the RNA was assessed with a Nanodrop-2000 (Thermo Fisher Scientific). RNasefree water was used to adjust the stock concentration of all RNA samples to $500 \mathrm{ng} / \mu \mathrm{L}$. Complementary DNA was synthesized from $1.5 \mu \mathrm{g}$ of total RNA using a High-Capacity cDNA Reverse Transcription Kit with genomic DNA remover (Abclonal Biotechnology, \#RK20403). In order to avoid the genomic DNA contamination, total RNA was treated with $4 \times$ gDNA remover mix t for $5 \mathrm{~min}$ at $37^{\circ} \mathrm{C}$, then for additional $5 \mathrm{~min}$ at $85{ }^{\circ} \mathrm{C}$, and stop at $4{ }^{\circ} \mathrm{C}$ in a $20 \mu \mathrm{L}$ reaction system. Then, total RNA with genomic DNA removal was treated with $5 \times \mathrm{RT}$ mix for $5 \mathrm{~min}$ at $25^{\circ} \mathrm{C}$, for $15 \mathrm{~min}$ at $42{ }^{\circ} \mathrm{C}$, for $5 \mathrm{~s}$ at $85{ }^{\circ} \mathrm{C}$, and stop at $4{ }^{\circ} \mathrm{C}$. All RT-qPCR products were amplified using a SYBR Green I Master Kit (Abclonal Biotechnology, \#RK21202) in $10 \mu \mathrm{L}$ reaction volume on an Applied Biosystems ${ }^{\circledR} 7500$ Real-Time PCR System. The reaction mix contains $5 \mu \mathrm{L}$ of $2 \times$ SYBR Green Fast mix, $3 \mu \mathrm{L}$ of RNase-free water and $0.5 \mu \mathrm{L}$ each of forward and reverse primer and $1 \mu \mathrm{L}$ of cDNA. The negative control for RT-qPCR used RNase-free water instead of cDNA template. The RT-qPCR reaction conditions were $95{ }^{\circ} \mathrm{C} 2 \mathrm{~min}$ followed by 40 cycles of $95{ }^{\circ} \mathrm{C}$ for $15 \mathrm{~s}, 55{ }^{\circ} \mathrm{C}$ for $15 \mathrm{~s}$ and $72{ }^{\circ} \mathrm{C} 30 \mathrm{~s}$. Then, the last cycle was $95{ }^{\circ} \mathrm{C}$ for $15 \mathrm{~s}, 60{ }^{\circ} \mathrm{C}$ for $15 \mathrm{~s}$ and $95{ }^{\circ} \mathrm{C}$ for $15 \mathrm{~s}$ for melt curve analysis. Each reaction had three replicates. The RT-qPCR products were confirmed by sequencing. All primers in the negative control group did not have any amplification. Expression levels were normalized to human glyceraldehyde 3-phosphate dehydrogenase gene $(G A P D H)$. The relative gene expression level is for target genes relative to reference gene. The gene expression level was calculated as $2(-\triangle \triangle \mathrm{Ct})$ method, and the $\triangle \mathrm{Ct}$ means the $\mathrm{Ct}$ of target gene minus the $\mathrm{Ct}$ of reference gene. The GAPDH forward primer was 5'-CGGAGTCAACGGATTTGGTC-3', and GAPDH reverse primer was 5'-TGGGTGGAATCATATTGG AACAT-3'. The POSTN forward primer was 5'-TGC CCAGCAGTTTTGCCCAT-3', and POSTN reverse primer was 5'-CGTTGCTCTCCAAACCTCTA-3'. The PENK forward primer was 5'-TAATGGAATGTGAAG GTAA-3', and PENK reverse primer was 5'-GGTTTG CTATTTTCTCTG-3'. The CDC25A forward primer was 5'-TGGAGGTGAAGAACAACA-3', and CDC25A reverse primer was $5^{\prime}$-GGTCAAGAGAATCAGAAT GG-3'.

\section{Statistical analysis}

A receiver operating characteristic (ROC) curve was drawn to calculate the area under the curve (AUC) to discriminate AR patients from normal controls in all AR GEO datasets. SPSS 16.0 for Windows (IBM, Chicago, USA) was used for ROC curve analyses, and other statistical analyses were performed using GraphPad Prism 7.0 software (GraphPad Software, La Jolla, CA).

\section{Results \\ Identification of DEGs in AR}

We performed a differential analysis of mRNAs and identified 997 DEGs in nasal epithelial cells from AR patients (n-DEGs), of which 559 were down-regulated and 438 were up-regulated $\left(\left|\log _{2} \mathrm{FC}\right|>1\right.$, adjusted $P$-value $<0.05)$. The same method was used to analyze and identify 165 DEGs (78 up-regulated and 87 downregulated) in Th2-enriched CD4+ T cells from peripheral blood samples (t-DEGs) in GSE75011 using the edge and limma packages in $\mathrm{R}$.

Volcano plots were used to display the gene expression data and $P$-value statistics of each dataset (Fig. 1A, B). We identified the top 50 genes that were significantly differentially expressed $\quad\left(\left|\log _{2} \mathrm{FC}\right|>1\right.$, adjusted $P$-value $<0.05)$ between AR patients and healthy controls in the heatmap (Fig. 1C, D).

\section{Pathway maps and GO functional enrichment in AR by MetaCore}

To explore the potential functions of the DEGs, we generated pathway maps and performed GO functional enrichment analysis in MetaCore $(P$-value $<0.05)$. The top ten significantly enriched pathways in AR (according to the pathway map) are shown in Fig. 2. The pathway map terms associated with epithelial cells in AR patients were related to chronic obstructive pulmonary disease (COPD) and asthma (Fig. 2A) (e.g., intercellular communication, the role of $\mathrm{CD} 8+\mathrm{Tc} 1$ cells in COPD, the interleukin-induced inflammatory response in asthmatic airway fibroblasts, the Th2 cytokine-induced expression of mucins, NF- $\mathrm{kB}$ AP-1 and MAPK-mediated proinflammatory cytokine production by eosinophils and the role of Th17 cells in asthma). On the other hand, NETosis in systemic lupus erythematosus (SLE), inhibition of TGF- $\beta 1$ signaling, IL-1 signaling, transcription androgen receptor nuclear signaling, and inhibition of RUNX3 signaling were the main enriched pathways in Th2-enriched CD4+ $\mathrm{T}$ cells from peripheral blood in AR patients (Fig. 2B).

The $\mathrm{n}$-DEGs in AR patients were also strongly associated with the GO BP terms multicellular organism development, anatomical structure development, and system development process (Fig. 3A), while t-DEGs in AR patients were mainly related to positive regulation 


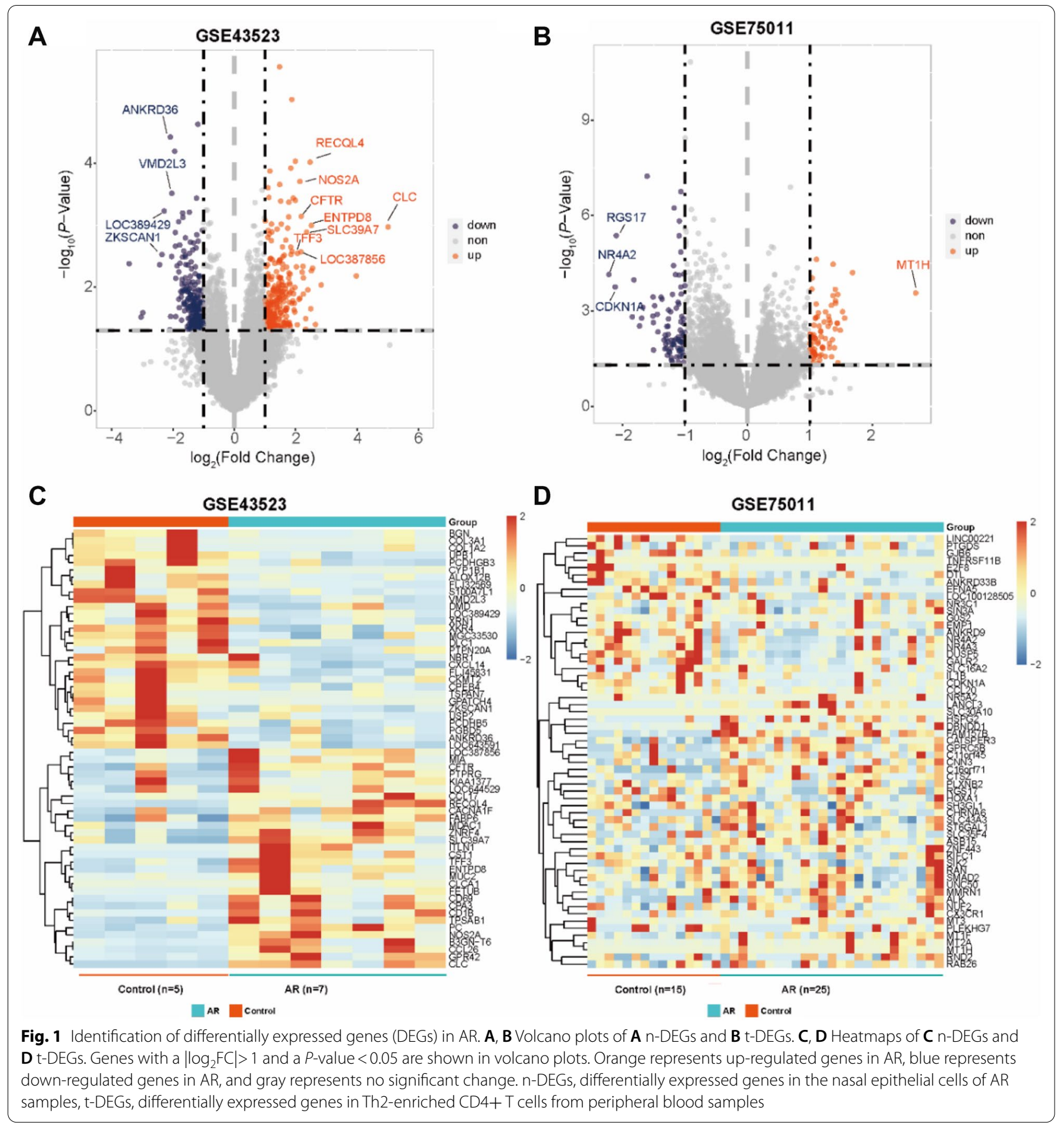

of nitrogen compound metabolic process, positive regulation of phosphorylation, and positive regulation of protein phosphorylation (Fig. 3B).

\section{PPI networks and hub DEGs in AR}

To illuminate the relationships between the DEGs and pathways, the ClueGO plugin of Cytoscape was used to perform GO functional enrichment and KEGG pathway analyses. Each node and line represent a term and the correlation between the terms, respectively. The color of the term shows the classification of nodes according to the functions. The results indicated that the n-DEGs were significantly enriched in axoneme, extracellular matrix, collagen fibril organization, cell motility, calcium 
A

GSE43523 Metacore maps

Inter-cellular relations in COPD (general schema) Interleukins-induced inflammatory response in asthmatic airway fibroblasts -

Th2-cytokines-induced expression of mucins in asthma

$\mathrm{NF}-\mathrm{kB}, \mathrm{AP}-1$ and MAPKs-mediated proinflammatory cytokine production by eosinophils in asthma

Role of CD8+ Tc1 cells in COPD -

Immune response_IL-13 signaling via JAK-STAT -

Role of fibroblasts and keratinocytes in the elicitation phase of allergic contact dermatitis

Dual function of Treg cells in cancer development -

Tumor-stroma interactions in pancreatic cancer - .

Role of Th17 cells in asthma - .

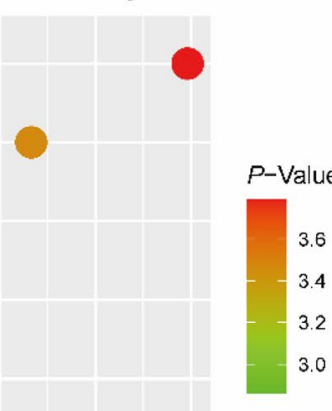

Counts

- 4.00

- 4.25

4.50

4.75

5.00

B

GSE75011 Metacore maps

NETosis in SLE.

Inhibition of TGF-beta 1 signaling in early colorectal cancer-

$\mathrm{IL}-1$ signaling in melanoma-

Transcription_Androgen Receptor nuclear signaling-

Inhibition of RUNX3 signaling in gastric cancer-
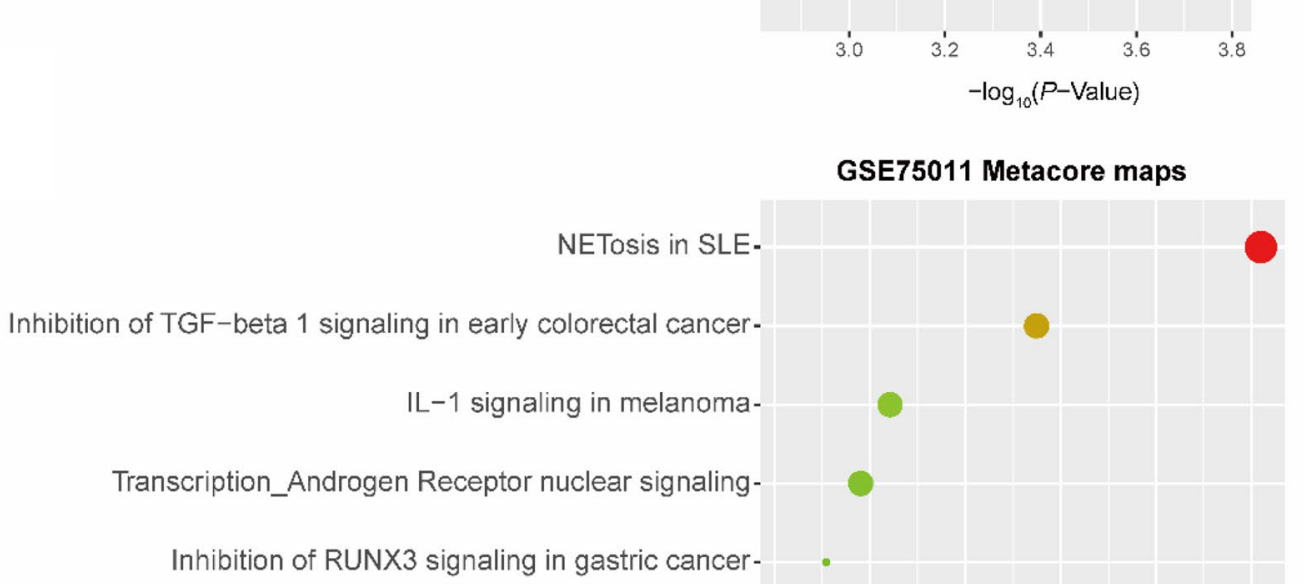

Disruption of apoptosis, proliferation and shedding of epithelial cells in asthma-

Transcription_HIF-1 targets-

Immune response_IL-5 signaling via JAK/STAT.

Immune response_Role of PKR in stress-induced antiviral cell response-

Cell cycle_Initiation of mitosis- .

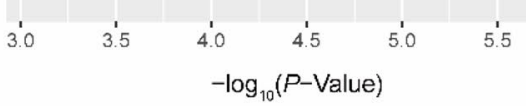

Fig. 2 Pathway maps of DEGs in AR. A, B Bubble chart showing pathway maps for $\mathbf{A} n$-DEGs and $\mathbf{B}$ t-DEGs by MetaCore 
A

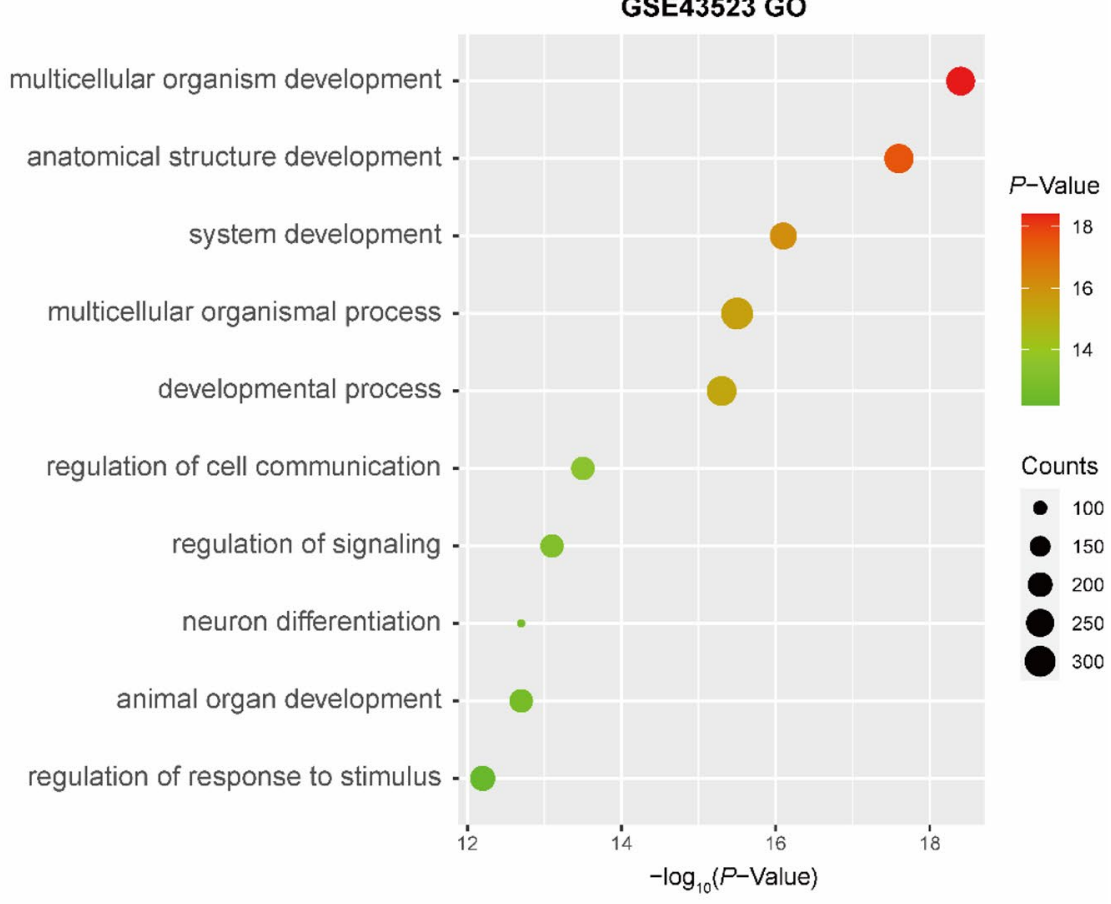

B

GSE75011 GO

positive regulation of nitrogen compound metabolic process positive regulation of phosphorylation positive regulation of protein phosphorylation positive regulation of biological process positive regulation of macromolecule metabolic process positive regulation of protein modification process. ounts cellular response to organic substancepositive regulation of phosphorus metabolic process -

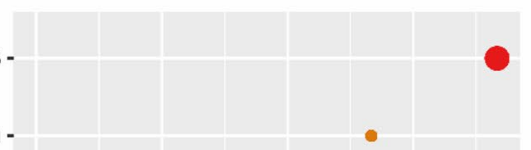
positive regulation of phosphate metabolic process positive regulation of metabolic process-

$$
11.4 \quad \begin{array}{lcc}
11.7 & 12.0 & 12.3 \\
& & -\log _{10}(P \text {-Value })
\end{array}
$$

Fig. 3 GO functional enrichment of DEGs in AR. A, B GO functional enrichment for A n-DEGs and $\mathbf{B}$ t-DEGs by MetaCore 


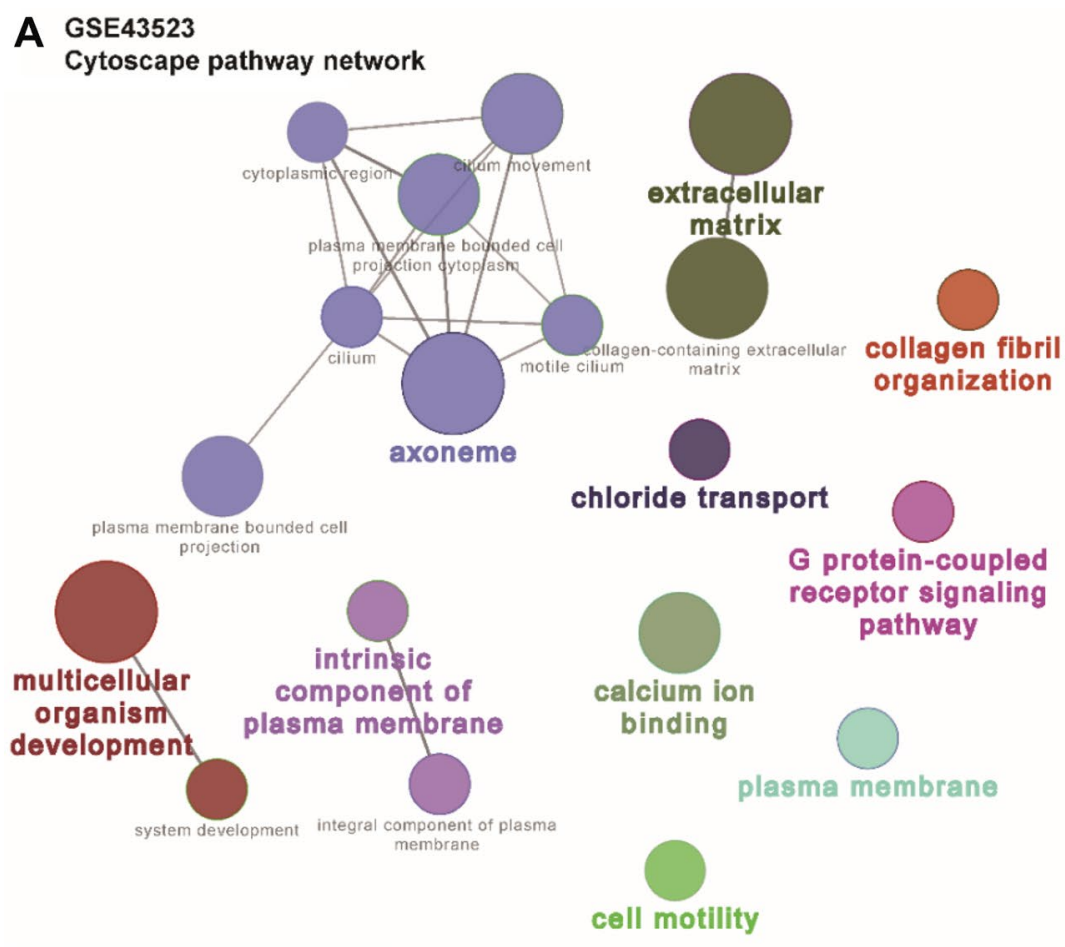

B GSE75011

Cytoscape pathway network

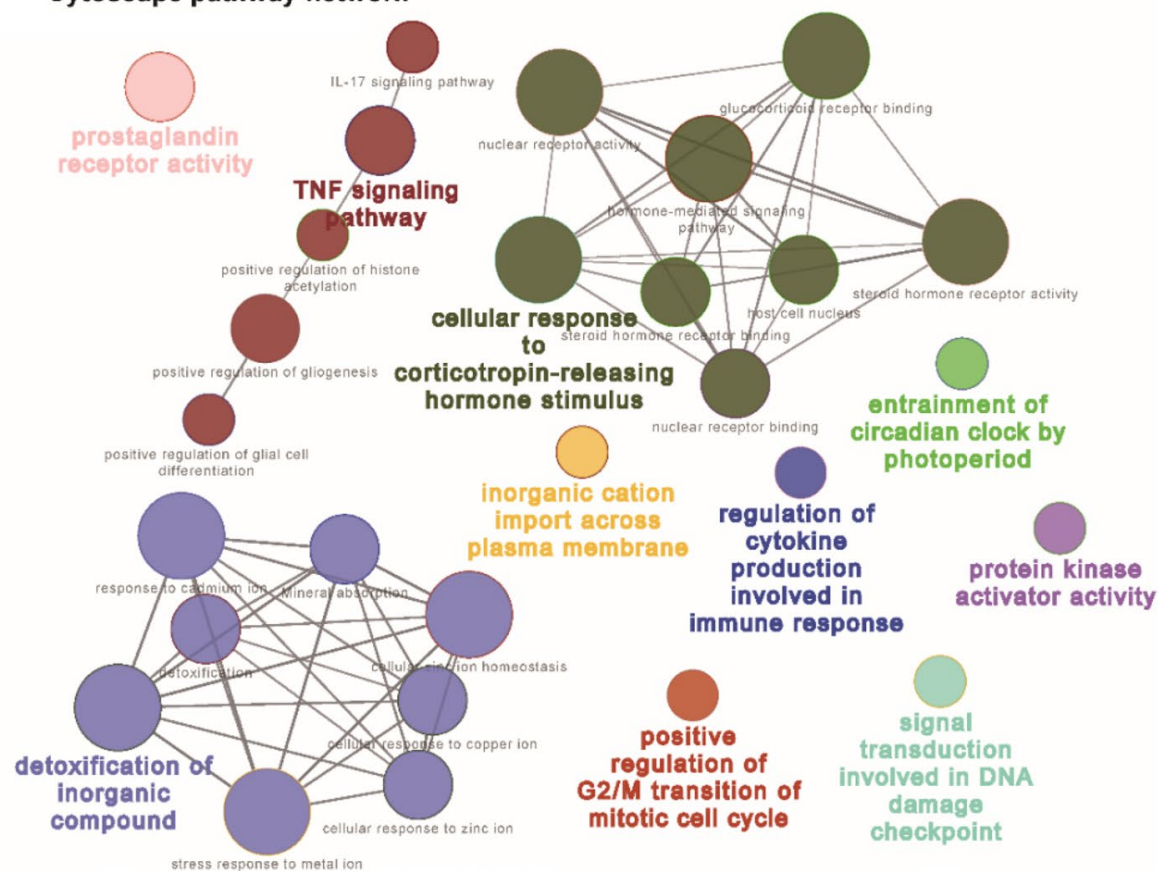

Fig. 4 PPI networks of DEGs in AR. A, B PPI networks for $\mathbf{A}$ n-DEGs and $\mathbf{B}$ t-DEGs. The node color represents the degree of proteins, and the edge color represents the combined score of proteins. Orange represents high, and blue represents low. C, D (C) n-DEGs and (D) t-DEGs in the PPI networks 


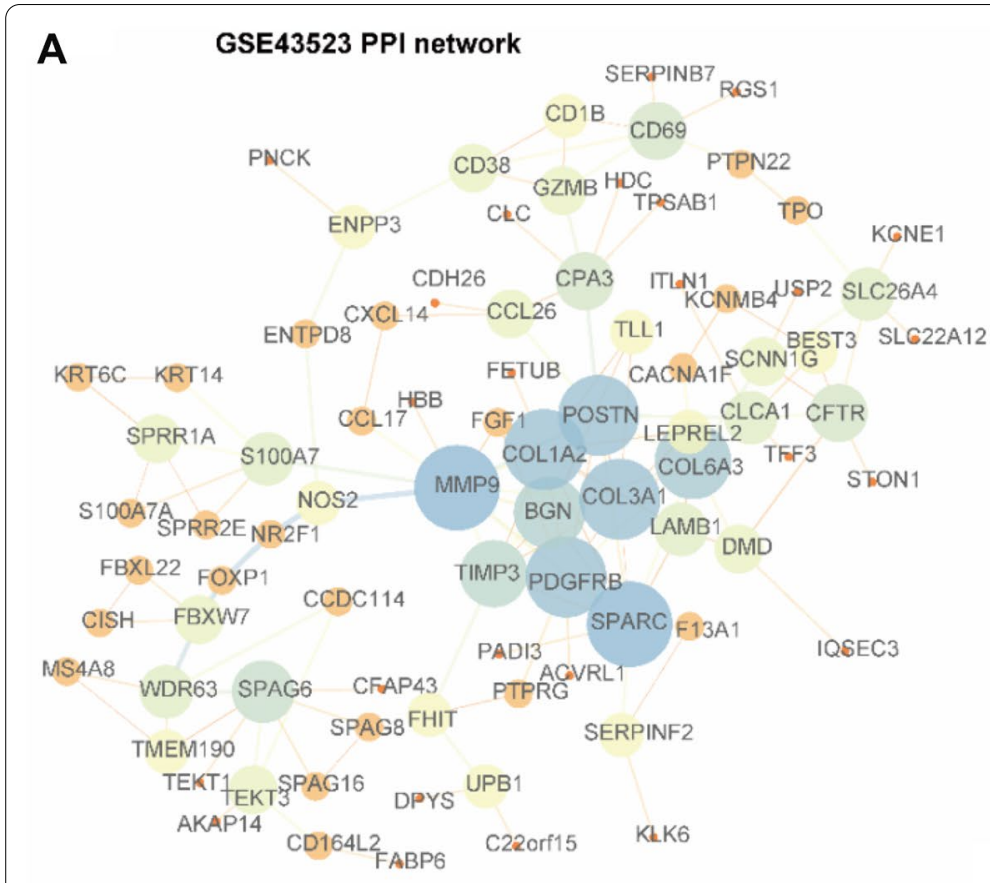

\section{GSE43523 hub genes}

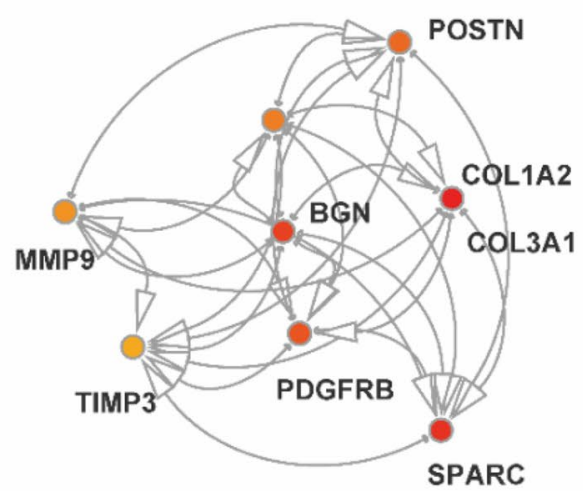

B

GSE75011 PPI network

D GSE75011 hub genes
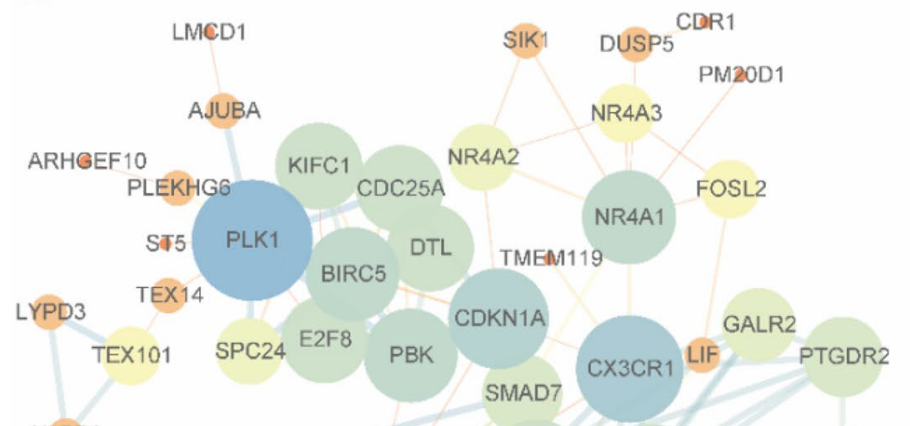

ULBP2

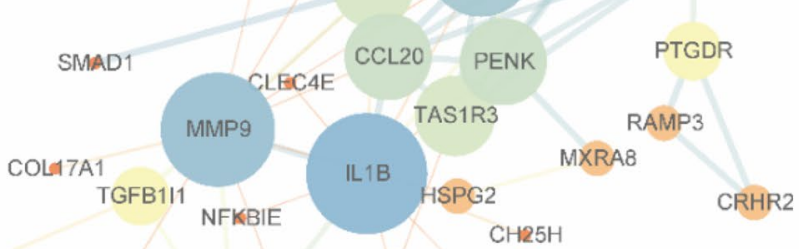

TRAF4
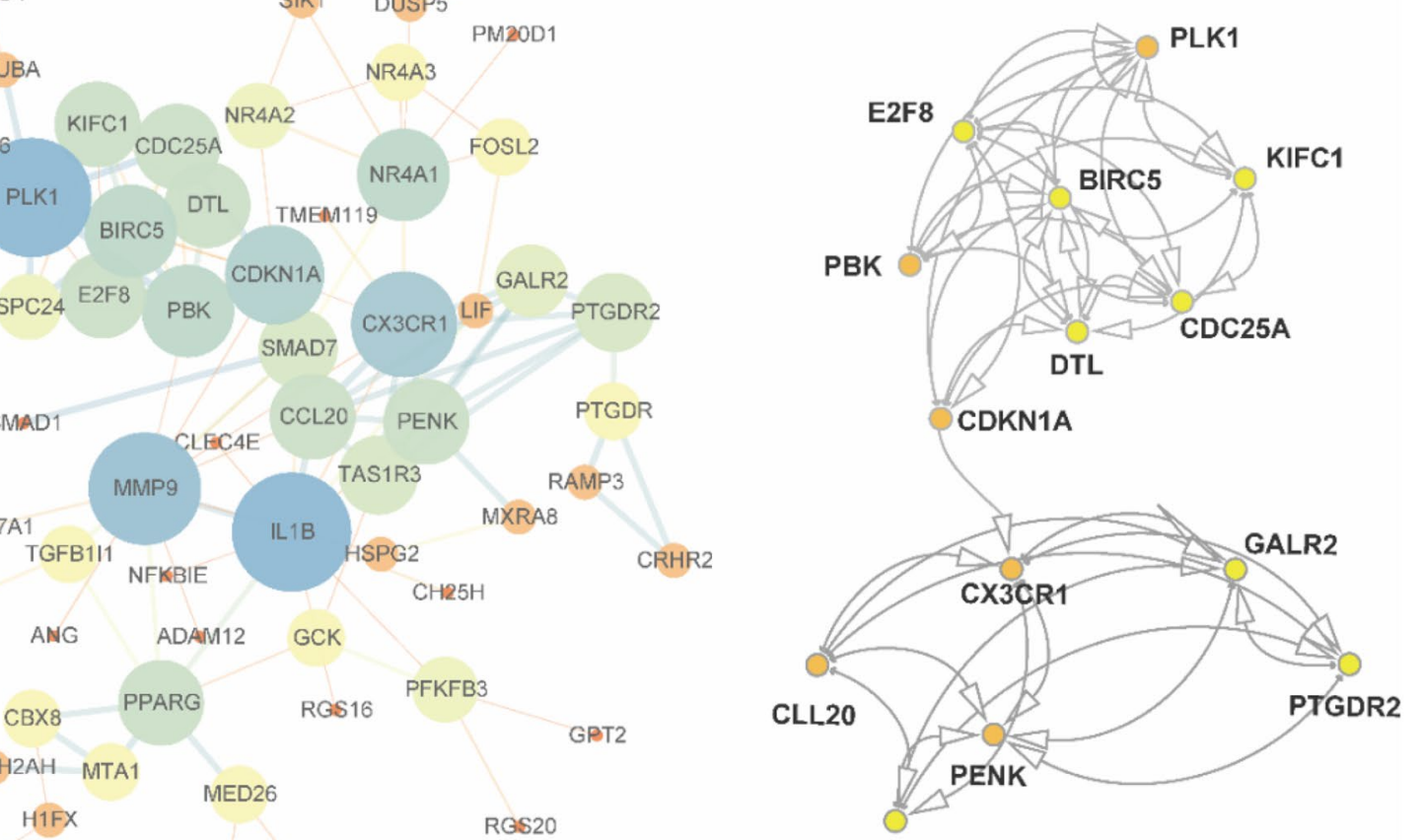

H3F3C

POLR2J2

NR2F6

Fig. 5 ClueGO for GO term and KEGG pathway analyses. A, B GO terms and KEGG pathways of $\mathbf{A} n-D E G s$ and $\mathbf{B}$ t-DEGs. Each node represents a GO term or KEGG pathway, and the size of the node is inversely associated with the P-value of enrichment. The color reflects the functional enrichment between the nodes, with the same function aggregating together in the same color. Genes with a kappa value $>0.4$ are shown in the diagram 
ion binding, and so on (Fig. 4A), while the t-DEGs were enriched in the TNF signaling pathway, detoxification of inorganic compound, and cellular response to corticotropin-releasing hormone stimulus (Fig. 4B).

To further explore the connections among the DEGs, PPI networks were constructed with the n-DEGs and t-DEGs. The PPI networks of n-DEGs contained 119 nodes and 160 edges, and the isolated genes (without interactions) were removed. A total of 145 interactions and 94 nodes were screened to establish the PPI networks of t-DEGs (Fig. 5A, B). The

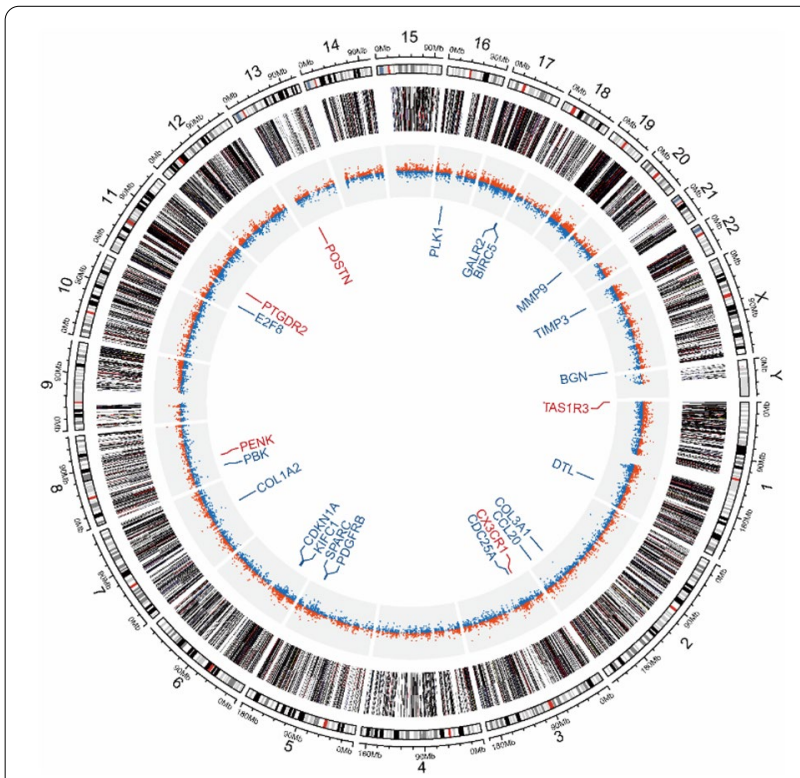

Fig. 6 Circos plots representing the distribution of DEGs in AR on human chromosomes. The outermost track of the Circos plot is the chromosome map of the human genome. Track 2 represents different gene biotypes of all DEGs from GSE43523 and GSE75011. Black, gray, red, orange, navy, purple, green, blue, yellow represent protein coding, long noncoding RNA (InCRNA), microRNA (miRNA), miscellaneous RNA (miscRNA), pseudogene, small nucleolar RNA (snoRNA), small nuclear RNA (snRNA), to be experimentally confirmed RNA (TEC), and other RNA, respectively. Track 3 represents the degree of difference between AR patients and healthy controls. The up-regulated or down-regulated genes are marked with red or blue plots, respectively, and plot heights in track 3 indicate the $\log _{2} \mathrm{FC}$ value of all DEGs from GSE43523 and GSE75011. Track 4 shows the labels of hub genes from the two GEO datasets. Red represents up-regulated genes, and blue represents down-regulated genes
MCODE algorithm was further applied to identify hub n-DEGs and t-DEGs that were densely associated with each other in the networks (Fig. 5C, D). We found 8 hub genes in the PPI networks of n-DEGs, namely, platelet-derived growth factor receptor $\beta$ (PDGFRB), biglycan $(B G N)$, secreted protein acidic and cysteine rich (SPARC), TIMP metallopeptidase inhibitor 3 (TIMP3), collagen type I $\alpha 2$ chain (COL1A2), collagen type III $\alpha 1$ chain (COL3A1), matrix metallopeptidase 9 (MMP9), and periostin (POSTN) and 14 hub t-DEGs, namely, E2F transcription factor 8 (E2F8), denticleless E3 ubiquitin protein ligase homolog $(D T L)$, kinesin family member C1 (KIFC1), proenkephalin (PENK), galanin receptor 2 (GALR2), taste 1 receptor member 3 (TAS1R3), prostaglandin D2 receptor 2 (PTGDR2), C-X3-C motif chemokine receptor 1 (CX3CR1), C-C motif chemokine ligand 20 (CCL20), cyclin dependent kinase inhibitor 1A (CDKN1A), PDZ binding kinase $(P B K)$, cell division cycle 25A (CDC25A), polo like kinase 1 (PLK1), and baculoviral IAP repeat containing 5 (BIRC5).

\section{Circular visualization of DEGs in AR}

A circular layout is an advantageous approach to present information along several chromosomes, multidimensional descriptions on the same chromosome, and relations between genomic rearrangements simultaneously. To obtain a better view of the symbols, chromosome locations, and expression levels of the DEGs, we drew Circos plots with the circlize package (Fig. 6). In Circos, each concentric ring is called a 'track'. First, the outermost track of the Circos plot was used to present the locations of chromosomes in the human genome assembly GRCh38 (hg38). Track 2 represents the different gene biotypes of all DEGs from the GSE43523 and GSE75011 datasets. Track 3 depicts the degree of difference between AR patients and healthy controls. The up-regulated or down-regulated genes are marked with red or blue plots, respectively, and plot heights in track 3 indicate the $\log _{2} \mathrm{FC}$ of all DEGs from the GSE43523 and GSE75011 datasets. To explore the locations of the hub genes from the two GEO datasets, track 4 was visualized, with red representing up-regulated genes and blue representing down-regulated genes. In general, chromosomes $2,3,5,6,8,11$, and 17 each contained 2 hub genes in AR. Different genes were proven to be relatively distributed on different chromosomes.

(See figure on next page.)

Fig. 7 The diagnostic values and expression level of 3 hub genes in AR nasal mucosa and in blood, respectively. A, B ROC curves (A) and normalized relative expression levels (B) of POSTN, PENK and CDC25A in AR from public GEO datasets. The AUC of POSTN, PENK and CDC25A were 0.789, 0.73 and 0.71. n-DEGs from combination of GSE101720, GSE19187, GSE46171 and GSE44037 databases for POSTN analysis. And t-DEGs from GSE50101 and GSE37157 for PENK and CDC25A analysis. C, D The relative expression levels (C) and ROC curves (D) of POSTN in nasal mucosa and PENK and CDC25A in blood of AR patients. The AUC of combined detection of the 3 indexes was 0.98 

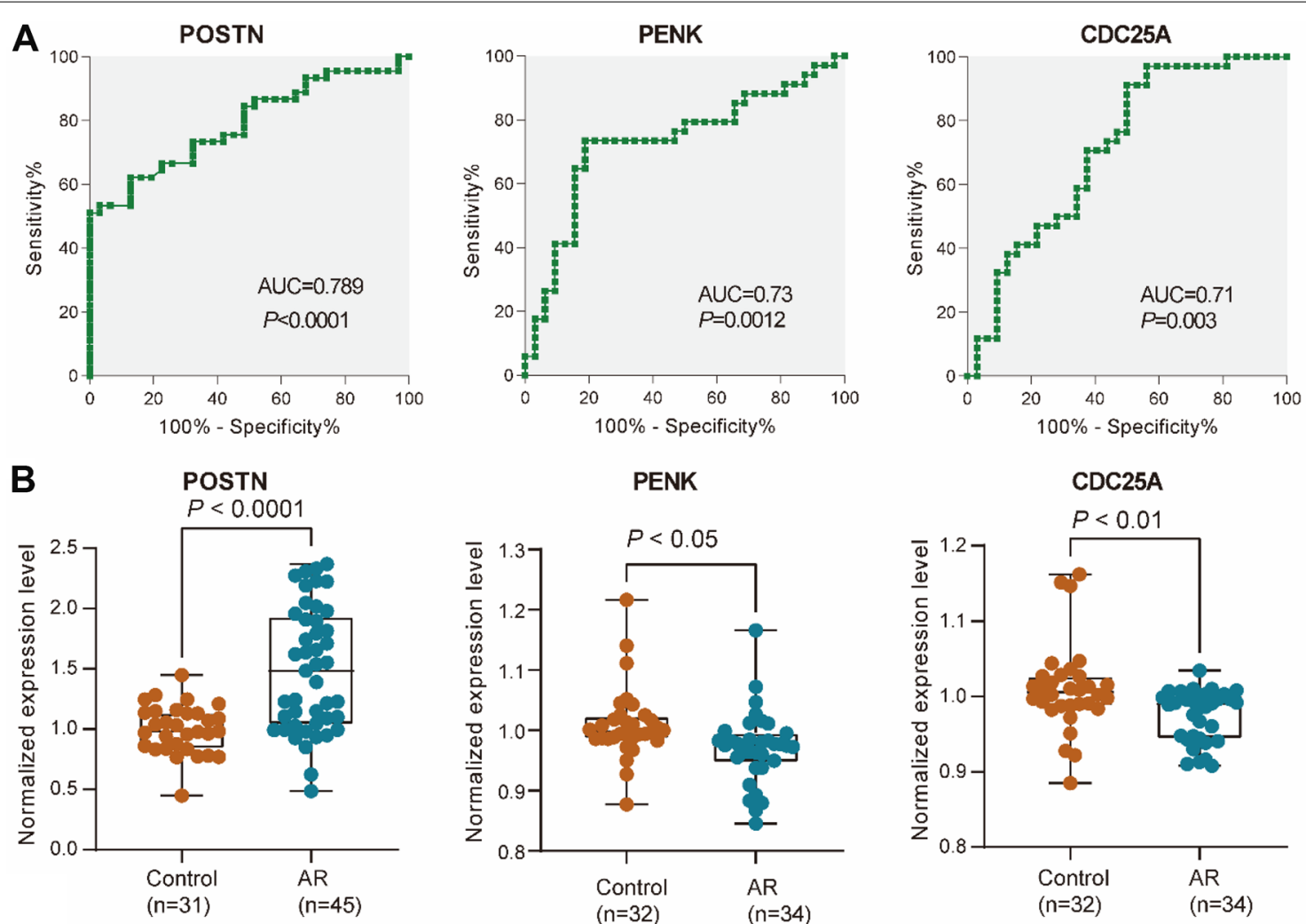

PENK
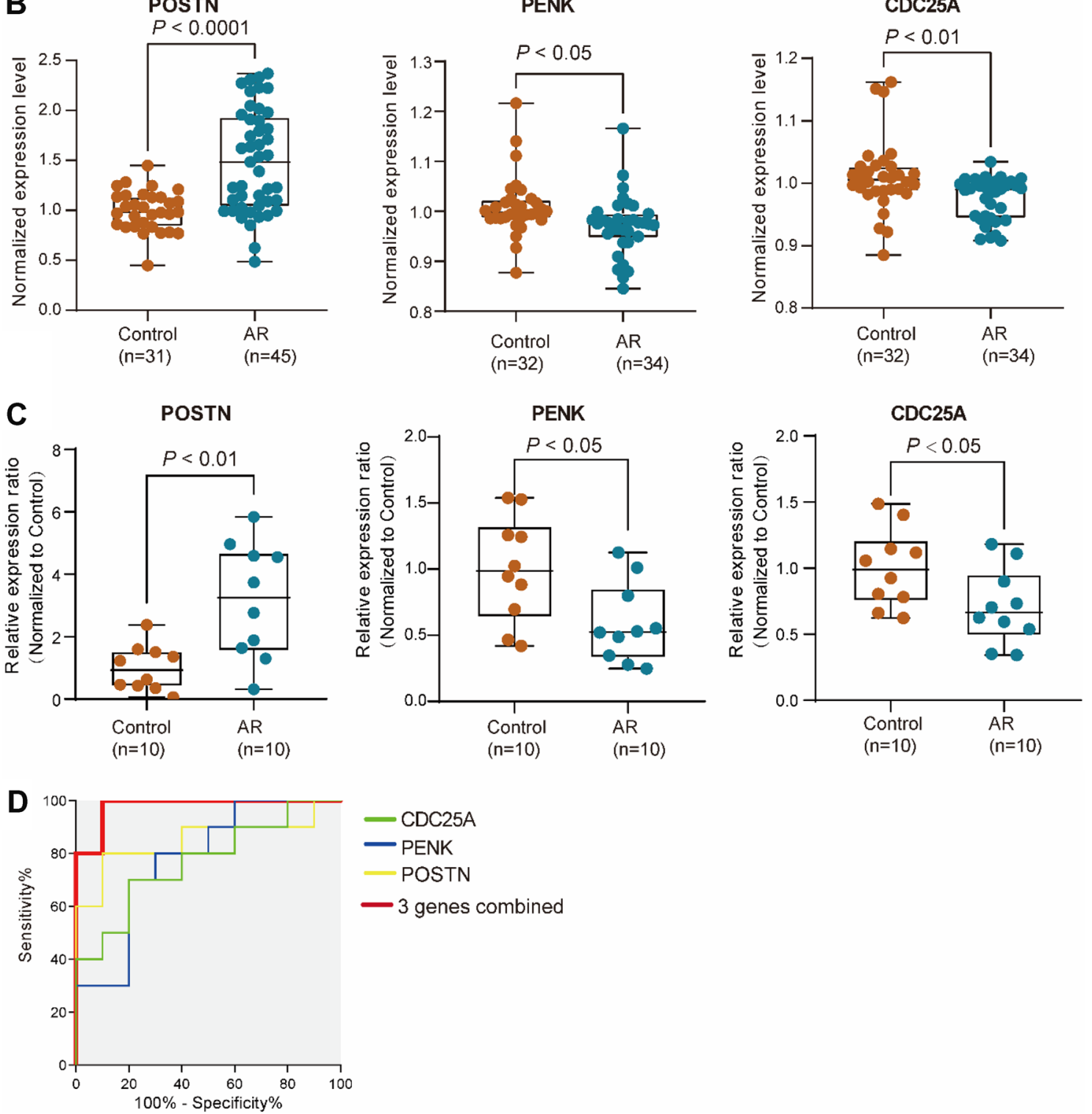

Fig. 7 (See legend on previous page.) 
Table 2 AUCs for predictors associated with AR

\begin{tabular}{llll}
\hline Gene & AUC & $\mathbf{9 5 \%} \mathrm{Cl}$ & $\boldsymbol{P}$ value \\
\hline POSTN & 0.85 & 0.663 to 1.037 & 0.0082 \\
PENK & 0.78 & 0.5727 to 0.9873 & 0.0343 \\
CDC25A & 0.77 & 0.5597 to 0.9803 & 0.0413 \\
3 gene combinations & 0.98 & 0.9295 to 1.031 & 0.0003 \\
\hline
\end{tabular}

$A U C$ area under the curve, $\mathrm{Cl}$ confidence interval

\section{Validation of hub genes based on GEO databases and RT-qPCR}

To validate the correlations between these hub genes and AR, We compared the expression of each candidate hub gene in nasal samples based on GSE101720, GSE19187, GSE46171 and GSE44037. In addition, GSE50101 and GSE37157 were used for validation of hub genes in blood. The ROC analysis revealed that only the expression levels of POSTN in nasal mucosa and PENK and CDC25A in blood showed excellent diagnostic value for AR diagnosis (Fig. 7A, B). Furthermore, RT-qPCR was performed to analyze the expression levels of POSTN in nasal mucosa and PENK and CDC25A in blood from the AR patients. We found that the expression level of POSTN was significantly increased in nasal mucosa, while the expression levels of PENK and CDC25A were decreased in blood of AR patients (Fig. 7C and Additional file 1: Tables S1, S2). The AUC of combined detection of the three indexes (POSTN, PENK and CDC25A) was 0.98 (Fig. 7D and Table 2). Taken together, our study demonstrated that the three candidate hub genes were significantly related to AR diagnosis.

\section{Discussion}

Our study was based on nasal epithelial cells and Th2enriched CD4+ cells from the peripheral blood of AR patients and nonallergic controls from the GEO database. We identified 997 n-DEGs (438 up-regulated and 559 down-regulated) from nasal epithelial cells and 165 t-DEGs (78 up-regulated and 87 down-regulated) from Th2-enriched CD4+ $\mathrm{T}$ cells. In addition, we identified 8 and 14 hub genes from PPI networks of the n-DEGs and t-DEGs that might be used as biomarkers in the pathogenesis of AR. Some of the hub genes have been studied in AR. MMP9, as a member of the matrix metalloproteinase family, has been demonstrated to act in a proinflammatory manner. The increased expression of MMP-9 in allergic nasal mucosa contributes to the migration of inflammatory cells to the nasal mucosa of AR patients [22, 23]. POSTN encodes a secreted extracellular matrix protein that functions in tissue development and regeneration and is upregulated in AR [24]. PTGDR2 is a prostaglandin D2 receptor that mediates the proinflammatory chemotaxis of eosinophils, basophils, and Th2 lymphocytes generated during allergic inflammation. PTGDR2 can also be used as a Th2-related gene to evaluate the effect of AR immunotherapy [25]. A study on nasal tissue from individuals with allergic and nonallergic chronic rhinosinusitis highlighted a novel role for $C X 3 C R 1$ (i.e., $C X 3 C R 1$ may contribute to natural killer cell trafficking to the allergic upper airway) [26]. The production of CCL20 in human nasal epithelial cells may increase after stimulation dsRNA [27], and the upregulation of CCL20 expression may contribute to the recruitment and retention of effector $\mathrm{T}$ cells in allergic asthma [28]. BGN, one of the small leucine-rich repeat proteoglycans involved in tissue remodeling in inflammatory diseases, showed strong immunoreactivity in mild and severe persistent allergic nasal mucosa [29].

In some previous studies, bioinformatic analysis of mRNAs and lncRNAs in AR was performed. For instance, two studies focused on DEGs from the nasal epithelial cells of AR patients [9, 30]. Another study focused on DEGs and the transcription factor (TF)miRNA network only in nasal epithelial cells and showed that miR-17-5P might act in SAR by targeting ATP binding cassette subfamily A member 1 (ABCA1) and CD69 [31]. Additionally, a study on DEGs in adult and pediatric nasal epithelial cells a found that AP-1 was associated with AR by regulating cystatin SN (CST1) and Charcot-Leyden crystal galectin (CLC) [32]. Circulating miR-125b, miR-16, miR-299-5p, miR-126, miR-206, and miR-133b in peripheral blood have been identified as predictive markers of allergic and asthmatic status [33]. A study based on Th2-enriched CD4+ $\mathrm{T}$ cells from peripheral blood samples revealed unique and common molecules that are likely to confer pathogenic features to Th2 cells in asthma and rhinitis [11]. These results together with the DEGs identified in different sources of samples suggest few intersecting genes obtained using different tissues or cells from different locations [34, 35]. Inherent gene expression due to potential confounders produced from highly expressed genes in different tissue or cell types might be ignored in an analysis of DEGs in a single source. Recently, some gene expression studies on asthma [36], musculoskeletal development [37], and nonalcoholic fatty liver disease [38] have reported approaches in which different tissues were combined.

In our study, MetaCore pathway map analysis showed that NETosis was the most significant differentially enriched pathway between AR patients and healthy controls. Neutrophil extracellular traps are the result of a unique form of cell death that is morphologically characterized by the loss of intracellular membranes before the integrity of the plasma membrane is compromised; this is called NETosis [39]. NETosis has been reported to be involved in asthmatic airway inflammation and chronic rhinosinusitis 
(CRS). A study on a mouse model of asthma showed that rhinovirus infection triggers dsDNA release associated with the inhibition of NETosis [40]. CRS displays variable degrees of eosinophilic and neutrophilic inflammation, with profound neutrophilic infiltration and activation in type 2 CRS with nasal polyps (CRSwNP) [41]. The underlying mechanisms of NETosis would be of considerable promise for AR patients. NF-kB-, AP-1- and MAPK-mediated proinflammatory cytokine production by eosinophils might play an important role in the nasal mucosa in AR. Similar to our study, a previous bioinformatic study found that AP-1 was associated with AR by regulating CST1 and CLC [32, 42]. IL-1 family cytokines (IL-1 $\beta$, IL-18, and IL-33), IL-25, and TNF superfamilies can activate the NF-kB and AP-1 pathways to initiate the production of IL-5 and IL-13 in allergic airway inflammation [43]. However, the roles of the NF- $\mathrm{kB}, \mathrm{AP}-1$ and MAPK pathways in AR still need to be completely explained. Chronic inflammation is a key factor that leads to tissue remodeling [44]. Whereas the epithelial to mesenchymal transition (EMT) process and remodeling have been proven to be characteristic of chronic airway inflammatory diseases such as asthma and CRS, the presence/absence and extent of tissue remodeling in the upper airways in AR are still controversial $[5,45,46]$. We also found that cell adhesion (extracellular matrix remodeling) and TGF- $\beta 1$ in fibrosis development are prominent significant pathways in the nasal epithelial cells of AR patients, suggesting that cell adhesion and tissue remodeling in the nasal mucosa of AR patients need to be explored in the future.

Although our study demonstrated that combination of multiple gene biomarkers had more accuracy to be diagnostic for AR, there are some major gaps exist in the AR biomarker space. In particular, our study is limited with the small sample size from the AR patients and healthy controls. It's better to include the data contains the progression of AR before and after treatment. Besides our study, additional work is needed to explore the underlying mechanisms that gene biomarkers involved.

\section{Conclusion}

In summary, our study aim to identify genes and pathways in the nasal epithelial cells and peripheral blood of AR patients through publicly available datasets. We identified the DEGs and analyzed their functions through GO analysis and PPI networks and identified the hub genes that might play crucial roles in the pathogenesis of AR. In addition, we validated the hub genes both by GEO database and RT-qPCR in nasal mucosa and in blood from AR patients and the healthy controls. Our study demonstrated that the increasing POSTN expression in nasal brushings, and the decreasing expressions of $C D C 25 A$ and PENK in blood in AR or their combination could be used to help AR diagnosis $(\mathrm{AUC}=0.98$ ). This study provides new insights into the overall mechanism of AR from the nasal cavity to the blood, but further research is needed to develop novel therapeutic strategies to drug discovery along with medical approaches to clinical treatment of AR.

\section{Supplementary Information}

The online version contains supplementary material available at https://doi. org/10.1186/s13223-022-00656-4.

Additional file 1: Figure S1. Flowchart of our study to identify potential gene markers in AR patients. Table S1. The raw mean Ct value of the nasal brush samples and the blood samples from AR patients and healthy controls. Table S2. The RNA concentration of the nasal brush samples and the blood samples from AR patients and healthy controls.

\section{Acknowledgements}

We thank the researchers who contributed to the GEO datasets and thank the patients and healthy volunteers who participated in this study.

\section{Authors' contributions}

$L Z$, and $Y Z$ conceived and designed the project; $Y H$ and $Y Z$ performed the bioinformatics analysis under LZ's supervision; BW and JZ downloaded the GSE datasheets and collected the clinical data; $Y H, J Z$ and $Y Z$ collected nasal brushing and blood samples; LZ, XW and YZ were responsible for the clinical assessments; $\mathrm{YH}$ and PW performed RNA isolation and RT-qPCR validation; $\mathrm{YH}$ and $\mathrm{YZ}$ wrote the manuscript. All authors read and approved the final manuscript.

\section{Funding}

This study was supported by the Program for the Changjiang Scholars and Innovative Research Team (IRT13082); the National Natural Science Foundation of China (81970852, 82000962 and 82171110); the Beijing Bai-Qian-Wan talent project (2019A32); the Public Welfare Development and Reform Pilot Project (2019-10), Beijing municipal science and technology project (Z181100001618002), and the CAMS Innovation Fund for Medical Sciences (2019-|2M-5-022).

\section{Availability of data and materials}

All data generated or analyzed during this study are included in this published article.

\section{Declarations}

Ethics approval and consent to participate

The study was performed in accordance with the Helsinki declaration and was approved by the Beijing TongRen Hospital Ethic Committee.

Consent for publication

All the study's participants signed a Written Informed Consent.

Competing interests

None of the authors have any conflict of interest in this work.

\section{Author details}

${ }^{1}$ Department of Otolaryngology Head and Neck Surgery and Department of Allergy, Beijing TongRen Hospital, Capital Medical University, Beijing 100730, China. ${ }^{2}$ Beijing Laboratory of Allergic Diseases and Beijing Key Laboratory of Nasal Diseases, Beijing Institute of Otolaryngology, Beijing 100005, China. ${ }^{3}$ Research Unit of Diagnosis and Treatment of Chronic Nasal Diseases, Chinese Academy of Medical Sciences, Beijing 100005, China. 
Received: 27 August 2021 Accepted: 3 February 2022

Published online: 04 March 2022

\section{References}

1. Wheatley LM, Togias A. Clinical practice. Allergic rhinitis. N Engl J Med. 2015;372:456-63.

2. Meltzer EO. Allergic rhinitis: burden of illness, quality of life, comorbidities, and control. Immunol Allergy Clin N Am. 2016;36:235-48.

3. Wang XD, Zheng M, Lou HF, Wang CS, Zhang Y, Bo MY, et al. An increased prevalence of self-reported allergic rhinitis in major Chinese cities from 2005 to 2011. Allergy. 2016:71:1170-80.

4. Bousquet J, Van Cauwenberge P, Khaltaev N, Aria Workshop G, World Health O. Allergic rhinitis and its impact on asthma. J Allergy Clin Immunol. 2001;108:S147-334.

5. Eifan AO, Durham SR. Pathogenesis of rhinitis. Clin Exp Allergy. 2016:46:1139-51.

6. Rondon C, Bogas G, Barrionuevo E, Blanca M, Torres MJ, Campo P. Nonallergic rhinitis and lower airway disease. Allergy. 2017;72:24-34

7. Goodwin S, McPherson JD, McCombie WR. Coming of age: ten years of next-generation sequencing technologies. Nat Rev Genet. 2016;17:333-51

8. Kulasingam V, Diamandis EP. Strategies for discovering novel cancer biomarkers through utilization of emerging technologies. Nat Clin Pract Oncol. 2008:5:588-99.

9. Song $Y$, Yan Z. Exploring of the molecular mechanism of rhinitis via bioinformatics methods. Mol Med Rep. 2018;17:3014-20.

10. Aguerri M, Calzada D, Montaner D, Mata M, Florido F, Quiralte J, et al. Differential gene-expression analysis defines a molecular pattern related to olive pollen allergy. J Biol Regul Homeost Agents. 2013;27:337-50.

11. Seumois G, Zapardiel-Gonzalo J, White B, Singh D, Schulten V, Dillon M, et al. Transcriptional profiling of Th2 cells identifies pathogenic features associated with asthma. J Immunol. 2016;197:655-64.

12. Mullol J, Valero A, Alobid I, Bartra J, Navarro AM, Chivato T, et al. Allergic rhinitis and its impact on asthma update (ARIA 2008). The perspective from Spain. J Investig Allergol Clin Immunol. 2008;18:327-34.

13. Ritchie ME, Phipson B, Wu D, Hu Y, Law CW, Shi W, et al. limma powers differential expression analyses for RNA-sequencing and microarray studies. Nucleic Acids Res. 2015;43:e47.

14. Wu Y, Zhang L, Zhang Y, Zhen Y, Liu S. Bioinformatics analysis to screen for critical genes between survived and nonsurvived patients with sepsis. Mol Med Rep. 2018;18:3737-43.

15. Yu G, Wang W, Wang X, Xu M, Zhang L, Ding L, et al. Network pharmacology-based strategy to investigate pharmacological mechanisms of Zuojinwan for treatment of gastritis. BMC Complement Altern Med. 2018;18:292.

16. Szklarczyk D, Franceschini A, Wyder S, Forslund K, Heller D, Huerta-Cepas J, et al. STRING v10: protein-protein interaction networks, integrated over the tree of life. Nucleic Acids Res. 2015;43:D447-52.

17. Shannon P, Markiel A, Ozier O, Baliga NS, Wang JT, Ramage D, et al. Cytoscape: a software environment for integrated models of biomolecular interaction networks. Genome Res. 2003:13:2498-504.

18. Mlecnik B, Galon J, Bindea G. Comprehensive functional analysis of large lists of genes and proteins. J Proteomics. 2018;171:2-10.

19. Bader GD, Hogue CW. An automated method for finding molecular complexes in large protein interaction networks. BMC Bioinform. 2003;4:2.

20. Bindea G, Mlecnik B, Hackl H, Charoentong P, Tosolini M, Kirilovsky A, et al. ClueGO: a Cytoscape plug-in to decipher functionally grouped gene ontology and pathway annotation networks. Bioinformatics. 2009:25:1091-3.

21. Gu Z, Gu L, Eils R, Schlesner M, Brors B. circlize Implements and enhances circular visualization in R. Bioinformatics. 2014:30:2811-2.

22. Mori S, Pawankar R, Ozu C, Nonaka M, Yagi T, Okubo K. Expression and roles of MMP-2, MMP-9, MMP-13, TIMP-1, and TIMP-2 in allergic nasal mucosa. Allergy Asthma Immunol Res. 2012;4:231-9.

23. Xu L, Cai Z, Yang F, Chen M. Activationinduced upregulation of MMP9 in mast cells is a positive feedback mediator for mast cell activation. Mol Med Rep. 2017:15:1759-64.
24. Ishida A, Ohta N, Suzuki Y, Kakehata S, Okubo K, Ikeda H, et al. Expression of pendrin and periostin in allergic rhinitis and chronic rhinosinusitis. Allergol Int. 2012;61:589-95.

25. Masieri S, Trabattoni D, Incorvaia C, De Luca MC, Dell'Albani I, Leo G, et al. A role for Waldeyer's ring in immunological response to allergens. Curr Med Res Opin. 2014;30:203-5

26. El-Shazly AE, Doloriert HC, Bisig B, Lefebvre PP, Delvenne P, Jacobs N. Novel cooperation between CX3CL1 and CCL26 inducing NK cell chemotaxis via CX3CR1: a possible mechanism for NK cell infiltration of the allergic nasal tissue. Clin Exp Allergy. 2013;43:322-31.

27. Xu G, Zhang L, Wang DY, Xu R, Liu Z, Han DM, et al. Opposing roles of $I L-17 A$ and IL-25 in the regulation of TSLP production in human nasal epithelial cells. Allergy. 2010;65:581-9.

28. Francis JN, Sabroe I, Lloyd CM, Durham SR, Till SJ. Elevated CCR6+ CD4+ T lymphocytes in tissue compared with blood and induction of CCL20 during the asthmatic late response. Clin Exp Immunol. 2008;152:440-7.

29. Kim TH, Lee JY, Lee HM, Lee SH, Cho WS, Ju YH, et al. Remodelling of nasal mucosa in mild and severe persistent allergic rhinitis with special reference to the distribution of collagen, proteoglycans, and lymphatic vessels. Clin Exp Allergy. 2010:40:1742-54.

30. Yan Z, Liu L, Jiao L, Wen X, Liu J, Wang N. Bioinformatics analysis and identification of underlying biomarkers potentially linking allergic rhinitis and asthma. Med Sci Monit. 2020;26:e924934.

31. Liu X, Ren Y, Sun X, Huang H, Liu X. Bioinformatics-based approaches predict that MIR-17-5P functions in the pathogenesis of seasonal allergic rhinitis through regulating $A B C A 1$ and CD69. Am J Rhinol Allergy. 2019:33:269-76.

32. Lei Y, Guo P, An J, Guo C, Lu F, Liu M. Identification of pathogenic genes and upstream regulators in allergic rhinitis. Int J Pediatr Otorhinolaryngol. 2018;115:97-103

33. Panganiban RP, Wang Y, Howrylak J, Chinchilli VM, Craig TJ, August A, et al. Circulating microRNAs as biomarkers in patients with allergic rhinitis and asthma. J Allergy Clin Immunol. 2016;137:1423-32.

34. Lage K, Hansen NT, Karlberg EO, Eklund AC, Roque FS, Donahoe PK, et al. A large-scale analysis of tissue-specific pathology and gene expression of human disease genes and complexes. Proc Natl Acad Sci USA. 2008;105:20870-5.

35. Reverter A, Ingham A, Dalrymple BP. Mining tissue specificity, gene connectivity and disease association to reveal a set of genes that modify the action of disease causing genes. BioData Min. 2008;1:8.

36. Ghosh D, Ding L, Bernstein JA, Mersha TB. The utility of resolving asthma molecular signatures using tissue-specific transcriptome data. G3 Genes Genomes Genet. 2020;10:4049-62.

37. Ferguson GB, Van Handel B, Bay M, Fiziev P, Org T, Lee S, et al. Mapping molecular landmarks of human skeletal ontogeny and pluripotent stem cell-derived articular chondrocytes. Nat Commun. 2018;9:3634.

38. Kurt Z, Barrere-Cain R, LaGuardia J, Mehrabian M, Pan C, Hui ST, et al. Tissue-specific pathways and networks underlying sexual dimorphism in non-alcoholic fatty liver disease. Biol Sex Differ. 2018:9:46.

39. Brinkmann $\vee$, Zychlinsky A. Neutrophil extracellular traps: is immunity the second function of chromatin? J Cell Biol. 2012:198:773-83.

40. Toussaint M, Jackson DJ, Swieboda D, Guedan A, Tsourouktsoglou TD, Ching YM, et al. Host DNA released by NETosis promotes rhinovirusinduced type-2 allergic asthma exacerbation. Nat Med. 2017;23:681-91.

41. Delemarre T, Holtappels G, De Ruyck N, Zhang N, Nauwynck H, Bachert C, et al. A substantial neutrophilic inflammation as regular part of severe type 2 chronic rhinosinusitis with nasal polyps. J Allergy Clin Immunol. 2020;147:179-88

42. Yang Y, Zhang Y, Yang Y, Guo J, Yang L, Li C, et al. Differential expression of long noncoding RNAs and their function-related mRNAs in the periphera blood of allergic rhinitis patients. Am J Rhinol Allergy. 2020;34:508-18.

43. Kato A. Group 2 innate lymphoid cells in airway diseases. Chest. 2019;156:141-9.

44. Van Bruaene N, Derycke L, Perez-Novo CA, Gevaert P, Holtappels G, De Ruyck N, et al. TGF-beta signaling and collagen deposition in chronic rhinosinusitis. J Allergy Clin Immunol. 2009;124:253-9.

45. Watelet JB, Van Zele T, Gjomarkaj M, Canonica GW, Dahlen SE, Fokkens W, et al. Tissue remodelling in upper airways: where is the link with lower airway remodelling? Allergy. 2006;61:1249-58. 
46. Eifan AO, Orban NT, Jacobson MR, Durham SR. Severe persistent allergic rhinitis. Inflammation but no histologic features of structural upper airway remodeling. Am J Respir Crit Care Med. 2015;192:1431-9.

\section{Publisher's Note}

Springer Nature remains neutral with regard to jurisdictional claims in published maps and institutional affiliations.

- fast, convenient online submission

- thorough peer review by experienced researchers in your field

- rapid publication on acceptance

- support for research data, including large and complex data types

- gold Open Access which fosters wider collaboration and increased citations

- maximum visibility for your research: over 100M website views per year

At BMC, research is always in progress.

Learn more biomedcentral.com/submissions 